\title{
Burden of care in primary caregivers of individuals with spinal cord injury in Iran: its association with sociodemographic factors
}

\author{
Z Khazaeipour ${ }^{1}$, F Rezaei-Motlagh ${ }^{1}$, E Ahmadipour ${ }^{1}$, M Azarnia-Ghavam ${ }^{1}$, A Mirzababaei ${ }^{1}$, N Salimi ${ }^{1}$ \\ and A Salehi-Nejad ${ }^{2}$
}

Study design: A descriptive cross-sectional study.

Objectives: The burden of care among primary caregivers of individuals with spinal cord injury (SCl) is affected by different factors. We aimed to evaluate the level of burden among caregivers and the association between the caregiver burden and sociodemographic factors of $\mathrm{SCl}$ individuals and their caregivers.

Setting: Brain and Spinal Cord Injury Research Center, Tehran University of Medical Sciences, Tehran, Iran.

Methods: The Zarit caregiver burden interview was used to evaluate the level of burden in caregivers through structured face-to-face interviews with 163 individuals with $\mathrm{SCl}$, who were referred to receive outpatient rehabilitation, and their caregivers.

Results: The mean of caregivers' burden score was $38.9 \pm 15.2$ and $11.7 \%$ reported no or little burden, $43.6 \%$ reported 'mild-tomoderate' burden, $33.1 \%$ reported 'moderate-to-severe' burden and $11.7 \%$ reported 'severe' burden of care. There was relationship between the level of injury $(P=0.010)$ and occupational status $(P=0.041)$ in $\mathrm{SCl}$ individuals and caregiver burden score. There was also a positive relationship between the caregiver burden score and duration of injury $(r=0.176, P=0.025)$, the caregivers' age $(r=0.350, P<0.001)$, and length of time that the current caregiver providing care $(r=0.253, P=0.001)$. There was a negative relationship between the burden and caregiver's educational level $(r=-0.235, P=0.002)$.

Conclusions: The burden of care among the caregivers of $\mathrm{SCl}$ individuals is a multidimensional issue. The sociodemographic characteristics of individuals with $\mathrm{SCl}$ and their caregivers can affect the caregiver's burden. Many of these factors can be modified to reduce the burden of care.

Spinal Cord (2017) 55, 595-600; doi:10.1038/sc.2016.195; published online 31 January 2017

\section{INTRODUCTION}

Spinal cord injury (SCI) is defined as damage to the spinal cord that results in the loss of motor, sensory and/or autonomic function. ${ }^{1,2}$ This condition causes numerous complications, including physical difficulties such as pain, pressures, ulcer and psychological distress like depression, ${ }^{3}$ most of which are accompanied with financial difficulties. ${ }^{4,5}$ The SCI not only markedly impacts those with the injury, but also their family members. The individuals with SCI dependent on others for their activities of daily life in different levels, ${ }^{6}$ and accordingly it challenges them and their families in terms of physical, social and psychological issues. ${ }^{1,7}$ Even though the backwash of SCI affects the entire family, typically one member is more involved as the primary caregiver. ${ }^{8}$

A primary or informal caregiver is a family member who is responsible for providing primary care for the patient ${ }^{2,6}$ and usually lives with them. ${ }^{9}$ Generally, primary caregivers do not have training in healthcare such as performing care for the patient or coping with post-injury emotional difficulties. ${ }^{6}$ It should be noted that the primary caregivers provide a wide range of services ${ }^{2,8,10}$ mostly without getting paid. $^{7}$ Previous studies indicate that the caregivers are markedly challenged and go through difficult circumstances throughout the care, ${ }^{8,11}$ which usually continues for a long time. ${ }^{12,13}$

It is worth noting that the care burden-particularly medical issues-faced by the caregivers of SCI individuals and the consequences of such difficulties may have an adverse impact on the patients' health-related quality of life. ${ }^{14}$ Hence, given that the caregiver is crucial to maintaining the patient, understanding, prevention and treatment of the care burden, particularly health-threatening problems, among caregivers may help to reduce the need for hospitalization of patients or, at least, increase the time between admissions. ${ }^{14}$

The burden of care can be defined as a multidimensional response to the negative appraisal and perceived stress resulting from taking care of the patient. ${ }^{15}$ Caregivers of SCI individuals, due to the unique challenges and long duration of the care, are at greater risk of physical, psychological and social difficulties in comparison with caregivers of other chronic patients. ${ }^{10}$ Various studies have reported an elevation in levels of physical stress, ${ }^{10}$ emotional

\footnotetext{
${ }^{1}$ Brain and Spinal Cord Injury Research Center, Neuroscience Institute, Tehran University of Medical Sciences, Tehran, Iran and ${ }^{2}$ Faculty of World Studies, University of Tehran, Tehran, Iran

Correspondence: Dr Z Khazaeipour, Brain and Spinal Cord Injury Research Center, Neuroscience Institute, Tehran University of Medical Sciences, Keshavarz Boulevard, Imam Khomeini Hospital Complex, Tehran 1419733141, Iran.

E-mail: khazaeipour@tums.ac.ir or z_kh14@yahoo.com

Received 7 September 2016; revised 19 December 2016; accepted 21 December 2016; published online 31 January 2017
} 
stress ${ }^{16}$ and isolation, ${ }^{9}$ which eventuate in burnout, fatigue, anger, ${ }^{10}$ depression, ${ }^{16}$ ill-health, dropping behind social and leisure activities, ${ }^{9}$ and financial difficulties ${ }^{16}$ in caregivers of SCI individuals. Therefore, the burden of the caregiver is a multidimensional concept ${ }^{8}$ which can be classified in two categories of objective and subjective burdens. The objective burdens are the outward consequences such as downturn of financial resources ${ }^{3}$ or disruption in family life and the activities related to provide care for SCI people, ${ }^{16}$ which are obviously visible to the others. The subjective burdens are the psychological consequences such as emotional stress or depression ${ }^{3}$ reported by caregiver based on personal appraisals of care-giving experience. ${ }^{17}$ Nevertheless, many studies have neglected the multidimensional nature of predictors for the caregiver burden and the ways in which multiple factors in the burden of care interact with each other. ${ }^{15}$

Considering the prominence of culture in influencing one's behavior and tolerance, and the estimation of one's burden of care, it should be noted that there is inadequate literature on the burden of the SCI caregivers in comparing with the prevalence of research on the other disease, ${ }^{14}$ particularly in the developing countries ${ }^{9}$ including Iran. Hence, understanding factors that influence the number of caregivers' burden and one's living cultural background, which are including geographical, historical and religious variable, are crucial to scientists to improve the lives of individuals with SCI and their caregivers.

Hence, this study aimed to evaluate the encumbrance of the caregivers of individuals with SCI and to assess the relation between sociodemographic variables and burden score in Iran.

\section{MATERIALS AND METHODS}

This cross-sectional study has been approved by the Ethics Committee of Tehran University of Medical Sciences. The participants were chosen among caregivers of those individuals with SCI who had been referred to the clinic of Brain and Spinal Cord Injury Research Center, Tehran University of Medical Sciences during 2015. The total number of 163 caregivers who (1) were a family member of the patient and had the primary responsibility for the patient's care, (2) at least 18 years old and (3) were unpaid caregivers participated in the study.

\section{Study methods}

The participants' perception of the study was justified and their written consent was obtained. They were also given reassurance concerning the confidentiality of their identity and information. The data were collected by trained interviewers that explained the questionnaire for caregivers to ensure their understanding of the questions and avoid confusion or misunderstanding. To avoid the bias concerning the care recipient's influence in answers, they had been kept separately in another room during the interviews.

The first part of the questionnaire touches upon demographic and socioeconomic details of SCI individuals and their caregivers including, sex, age, education, marital and occupational status, and kinship between the caregiver and recipient, and whether they live together or not. Injury characteristics of SCI people such as completeness, level of injury, etiology and duration of injury were also asked about in this part. We also asked inquired the accommodation condition (personal or rental housing) as an indicator of financial status of participants.

\section{Measurement}

For the next part, we used the Zarit caregiver burden interview (ZBI), which was translated, validated and proved by a panel of experts for assessing the subjective burdens including the role and personal burden, in 22 questions. Each item is scored on a 5-point scale from 0 to 4 : never (0), rarely (1), sometimes (2), quite frequently (3) and almost always (4); with the total score of 88 , as which the higher scores indicate greater burden for the caregivers of SCI individuals. Based on the participants' scores of ZBI, they were categorized
Table $1 \mathrm{SCl}$ individuals' and caregivers' characteristics

SCl individuals' characteristics

\begin{tabular}{lc}
\hline Age (years) & $36.04 \pm 12.54$ \\
Age at the time of injury (years) & $29.65 \pm 13.07$ \\
Duration of SCl (month) & $76.51 \pm 79.09$ \\
Educational level (years) & $8.39 \pm 4.65$ \\
& \\
Occupation & $19(11.8 \%)$ \\
Employed & $135(83.9 \%)$ \\
Unemployed & $7(4.3 \%)$ \\
Student & \\
Marital status & \\
Married & $92(56.4 \%)$ \\
Single & $62(38 \%)$ \\
Divorced & $8(4.9 \%)$ \\
Widow & $1(0.6 \%)$ \\
Sex & \\
Male & $131(80.4 \%)$ \\
Female & $33(19.6 \%)$ \\
Level of injury & \\
Paraplegia & \\
Tetraplegia & $127(79.9 \%)$ \\
Completeness & $32(20.1 \%)$ \\
Incomplete & \\
Complete & $70(51.1 \%)$ \\
Causes of SCl & $67(48.9 \%)$ \\
Crash injury & \\
Falling & \\
Occupational injury & \\
Non-traumatic & $88(54.3 \%)$ \\
latrogenic & $29(17.9 \%)$ \\
Sport injury & $21(13.0 \%)$ \\
Fight & $9(5.6 \%)$ \\
\end{tabular}

\section{Caregivers' characteristics}

Age (years)

$38.10 \pm 13.27$

Duration of giving care (month)

$69.44+73.13$

Educational level (years)

$8.77 \pm 4.79$

Sex

Male

$61(37.4 \%)$

Female

$102(62.6 \%)$

Occupation

Employed

Unemployed

$51(31.3 \%)$

$97(59.5 \%)$

$15(9.2 \%)$

Being Only caregiver

Yes

$87(53.4 \%)$

$76(46.6 \%)$

Living with $\mathrm{SCl}$ individual

Yes

$128(78.5 \%)$

No

$35(21.5 \%)$

Kinship

Parents

Spouse

Other member of family

$38(23.3 \%)$

$46(28.2 \%)$

$79(48.5 \%)$

Abbreviation: $\mathrm{SCl}$, spinal cord injury.

into four groups representing different severity levels of burden. Scores in the range of 0-20 were classified as 'little or no burden', scores 21-40 represented 'mild-to-moderate burden', range 41-60 was categorized as 'moderate-to-severe burden' and finally scores between 61 and 88 were classified as 'severe burden'. The reliability (internal consistency) of the test was assessed by Cronbach's $\alpha(0.876)$.

We added seven questions at the end of ZBI. By extending the questionnaire and adding our own developed questions to the original questionnaire, we tried to give a clearer point of view to our participants. We believe that 
Table 2 Additional questions about caregiver burden

\begin{tabular}{|c|c|c|c|c|c|}
\hline Questions & Never & Rarely & Sometimes & Nearly always & Always \\
\hline (1) Have you ever felt despaired or depressed in coping with the problems of the patient? & $31(19.01 \%)$ & $39(23.92 \%)$ & $57(34.96 \%)$ & $12(7.36 \%)$ & $24(14.72 \%)$ \\
\hline (2) Do you have any sleeping disorder that could be due to the care? & $66(40.49 \%)$ & $29(17.79 \%)$ & $32(19.63 \%)$ & $9(5.52 \%)$ & $27(16.56 \%)$ \\
\hline (3) Has physical elements of your living place ever been a problem for the patient care? & $70(42.94 \%)$ & $33(20.24 \%)$ & $28(17.17 \%)$ & $7(4.29 \%)$ & $25(15.33 \%)$ \\
\hline $\begin{array}{l}\text { (4) Do you sometimes feel that you want to run away from all the problems and the current } \\
\text { circumstances? }\end{array}$ & $103(63.19 \%)$ & $17(10.42 \%)$ & $19(11.65 \%)$ & $4(2.45 \%)$ & $20(12.26 \%)$ \\
\hline (5) Does the patient care impair your plans for the future? & $33(20.24 \%)$ & $27(16.56 \%)$ & $42(25.76 \%)$ & $18(11.04 \%)$ & $43(26.38 \%)$ \\
\hline (6) Does the patient care cause financial costs for you? & $5(3.06 \%)$ & $6(3.68 \%)$ & $25(15.33 \%)$ & $31(19.01 \%)$ & $96(58.89 \%)$ \\
\hline (7) Have you ever tried to provide care for psychological needs of the patient? & $4(2.45 \%)$ & $24(14.72 \%)$ & $54(33.12 \%)$ & $39(23.92 \%)$ & $42(25.76 \%)$ \\
\hline
\end{tabular}

additional questions regarding the real components of daily life, rather than merely abstract and conceptual concepts, assisted participants to reach a more comprehensive conclusion and improve the accuracy of the answers. As an illustration, inquiring about the quality of sleeping, progress of their initiative, and quality of their home and leisure time has clearer meanings for people. Nevertheless, we decided to keep the score of the additional questions separated from the original score of the participants and merely consider them as suggestions to enhance the questionnaire and its reliability. Finally, reliability of the questionnaire, which consists of 29 questions (ZBI and the additional questions), indicated improvement in internal consistency and accuracy. (Cronbach's $\alpha$ : 0.912).

We inquired the total years of education to obtain the educational level of the participants; as certain degrees may have different equivalents in different countries, the total years of education may prevent from such misinterpretation.

The participants were inquired regarding their residential accommodations as a measurement for their financial status. As for average people in Iran, ownership of a residential accommodation is a serious investment, which in turn lifts the heavy load of rental expenditures. We found that clustering participants based on their accommodation ownership was more reliable than their gross income range that undergoes different fluctuations.

The participants with personal housing were classified as those with good economic status and others who lived in rented accommodation were classified as disadvantaged economic status.

Medical information of SCI individuals, comprising their level of injury and completeness of injury, was collected from their medical records in Brain and Spinal Cord Injury Research Center clinic.

\section{Statistical analysis}

All collected data were analyzed by SPSS Inc (Chicago, IL, USA) version 18.0. In addition, the correlation of continuous variables was assessed by Pearson correlation. T-test for comparing means of continuous variables in two categorical variables and analysis of variance for comparing means of continuous variables in more than two categorical variables were used. The least significant difference was used for post hoc comparison. The linear regression analysis has been employed to evaluate the relationship among the caregivers burden score (dependent variable) and the duration of SCI, the caregivers' level of education and the caregivers' age (independent variables). Continuous variables were demonstrated as mean \pm s.d. and categorical variables were presented as frequency (percent). $P$-value of 0.05 was set as significance level.

\section{RESULTS}

The research was conducted with the enrollment and contribution of 163 out of 189 , which indicates the response rate of $86.2 \%$. The sociodemographic details of SCI individuals and their caregivers, in addition to the SCI injury characteristics, are demonstrated in Table 1.

Occupational status showed $105(65.2 \%)$ of SCI individuals were employed before the injury, whereas only 19 (11.8\%) had sustained a job after the injury.

The frequency of responses to the seven supplementary questions at the end of the ZBI is illustrated in Table 2.
Table 3 Association of caregivers' burden score and continuous variables

\begin{tabular}{lcr}
\hline & \multicolumn{2}{c}{ Caregiver burden score } \\
\cline { 2 - 3 } & \multicolumn{1}{c}{$r$} & P-value \\
\hline SCl individual age & \multicolumn{1}{c}{0.102} & 0.195 \\
Age at time of injury & 0.006 & 0.939 \\
Duration of injury (month) & $0.176^{*}$ & 0.025 \\
Education level of SCl individual & 0.035 & 0.654 \\
The number of children of SCl individual & 0.001 & 0.992 \\
Caregiver age & $0.350^{* *}$ & $<0.001$ \\
Caregiver education & $-0.235^{* *}$ & 0.002 \\
Care-giving duration of care & $0.253^{* *}$ & 0.001 \\
\hline
\end{tabular}

\section{$* P<0.05$.}

${ }^{* *} P<0.01$

Finally, only 19 (11.7\%) among 163 caregivers reported no or little burden, whereas $71(43.6 \%)$ of them described a mild-to-moderate burden, $54(33.1 \%)$ participants reported moderate-to-severe burden and $19(11.7 \%)$ of them reported severe burden of care. The relationship between the burden score, and the continuous variables (Table 3) and categorical variables (Table 4) was assessed as demonstrated in Tables 3 and 4.

\section{Caregiver burden score and SCI individual characteristics}

There was no relationship between the burden score and SCI individuals' age, sex or level of education. However, there was a relationship between the burden score and the occupational status of SCI people after their injury; their caregivers' burden was lower when they had a job $(P=0.041)$. The results regarding the injury characteristics indicated a relationship between caregiver's burden and the level of injury $(P=0.010)$; the caregivers of tetraplegic people felt more burden than the caregivers of individuals with paraplegia injury. There was also a positive relationship between the care burden score and duration of injury; and a longer duration of injury leads to a higher caregiver burden score $(r=0.176, P=0.025)$.

\section{Caregiver burden score and caregiver characteristics}

There was no relationship between sex of the caregiver and burden score. However, the caregivers' age had a direct effect on the burden score $(r=0.350, P<0.001)$ and older caregivers reported higher burden of care. There was a negative relationship between the burden score, and caregiver's educational level $(r=-0.235, P=0.002)$, the higher education level and the lower caregiver burden score. Although no relationship was observed between the occupational status of the 
Table 4 Association of caregivers' burden score with categorical variables

\begin{tabular}{|c|c|c|c|}
\hline & N & $\begin{array}{l}\text { Caregivers' burden score } \\
\qquad(\text { mean } \pm \text { s.d.) }\end{array}$ & P-value \\
\hline \multicolumn{4}{|c|}{ SCl individuals sex } \\
\hline Male & $131(80.4 \%)$ & $38.57 \pm 14.66$ & $0.622^{\mathrm{a}}$ \\
\hline Female & $32(19.6 \%)$ & $40.06 \pm 17.65$ & \\
\hline \multicolumn{4}{|l|}{ Level of Injury } \\
\hline Paraplegia & $127(79.9 \%)$ & $37.31 \pm 14.71$ & $0.010^{a}$ \\
\hline Tetraplegia & $32(20.1 \%)$ & $45.06 \pm 16.44$ & \\
\hline \multicolumn{4}{|c|}{ Completeness of injury } \\
\hline Incomplete & $70(51.1 \%)$ & $38.25 \pm 16.89$ & $0.742^{\mathrm{a}}$ \\
\hline Complete & $67(48.9 \%)$ & $39.15 \pm 14.56$ & \\
\hline \multicolumn{4}{|c|}{ Marital status of $\mathrm{SCl}$ individual } \\
\hline Single & $62(38 \%)$ & $40.92 \pm 15.63$ & $0.149^{b}$ \\
\hline Married & $92(56.4 \%)$ & $36.93 \pm 14.33$ & \\
\hline Divorced & $8(4.9 \%)$ & $44.44 \pm 19.87$ & \\
\hline \multicolumn{4}{|c|}{ SCl individuals' occupation after injury } \\
\hline Unemployed & $135(83.9 \%)$ & $40.14 \pm 15.07$ & $0.041^{b, c}$ \\
\hline Employed & $19(11.8 \%)$ & $32.74 \pm 15.39$ & \\
\hline Student & $7(4.3 \%)$ & $30.00 \pm 13.69$ & \\
\hline \multicolumn{4}{|l|}{ Caregiver sex } \\
\hline Male & $61(37.4 \%)$ & $36.65 \pm 11.69$ & $0.118^{a}$ \\
\hline Female & $102(62.6 \%)$ & $40.18 \pm 16.93$ & \\
\hline \multicolumn{4}{|c|}{ Caregiver occupation } \\
\hline Unemployed & 97 (59.5\%) & $41.05 \pm 41.05$ & $0.073^{b}$ \\
\hline Employed & $51(31.3 \%)$ & $36.19 \pm 11.19$ & \\
\hline Student & $15(9.2 \%)$ & $33.80 \pm 13.60$ & \\
\hline \multicolumn{4}{|c|}{ Are you just the person that gives care to patient? } \\
\hline Yes & $87(53.4 \%)$ & $40.05 \pm 15.85$ & $0.291^{\mathrm{a}}$ \\
\hline No & $76(46.6 \%)$ & $37.51 \pm 14.49$ & \\
\hline \multicolumn{4}{|c|}{ Do you live whit patient? } \\
\hline Yes & $128(78.5 \%)$ & $40.05 \pm 15.19$ & $0.058^{a}$ \\
\hline No & $35(21.5 \%)$ & $34.54 \pm 14.84$ & \\
\hline \multicolumn{4}{|c|}{ Who was the caregiver? } \\
\hline Parents & $38(23.3 \%)$ & $46.89 \pm 15.97$ & $<0.001^{\mathrm{b}, \mathrm{d}}$ \\
\hline Spouse & $46(28.2 \%)$ & $38.48 \pm 14.96$ & \\
\hline $\begin{array}{l}\text { Other member } \\
\text { of family }\end{array}$ & $79(48.5 \%)$ & $35.23 \pm 13.69$ & \\
\hline
\end{tabular}

Abbreviations: ANOVA, analysis of variance; $\mathrm{SCl}$, spinal cord injury.

a $T$-test.

bANOVA.

'Pair-wise comparison showed the difference between unemployed and employed group

$(P=0.047)$.

dPair-wise comparison showed the difference between parents and spouse $(P=0.009)$ and parents and other member of family $(P<0.001)$.

caregivers and their burden. In addition, the length of the care for SCI individuals provided by the current caregiver had a positive relationship with the care burden $(r=0.253, P=0.001)$. In other words, the caregivers, who provided care for a longer period of time, had higher burden scores. In the case of kinship of the caregivers and the care recipients and its relationship with the burden score, the post hoc test revealed greater burden in parents in comparison with spouses or other family members $(P<0.001)$. A tendency towards this significant relationship and escalated burden of care was for the caregivers who lived with the care recipients $(P=0.058)$. We analyzed the caregiver burden score in separate groups of the parents, spouses and other member of the family based on their living status with SCI individuals. The results showed that almost all of the parents (37/38, 97.4\%), and spouses $(44 / 46,95.6 \%)$, lived with the SCI individuals. Hence, we could not separate these two groups based on living with or not living with SCI individuals. However, approximately half of the other members of the family $(47 / 79,59.5 \%)$ happen to live with SCI persons. Analysis showed that there was no significant difference between the caregiver score means in the two groups. Nevertheless, there was no relationship between the burden score and being the only person who provides care for SCI individual (Table 4). The linear regression analysis showed that of the duration of SCI, the caregivers' level of education and the caregivers' age (independent variables), only the caregiver age had an independent association with caregiver burden score $(\beta=3.34, P=0.001)$.

\section{DISCUSSION}

In this study, the mean of caregivers' burden score was $38.86 \pm 15.25$ and the majority of the caregivers reported 'mild-to-moderate' burden. The result was the same as a similar study conducted in Iran, ${ }^{16}$ in which most of the participants as the caregivers of SCI individuals reported 'mild-to-moderate' burden. In another similar study conducted in China, Hai-Ping Ma et al. ${ }^{18}$ reported moderate-tosevere burden among $88 \%$ of primary caregivers, and their mean score of Zarit caregiver burden interview (22-Q) was $52.9 \pm 11.6$, which was considerably higher than the mean score in the current study. On the other hand, a study reported a mean burden of $11.8 \pm 7.7$ that is noticeably lower than results from this study. ${ }^{12}$ This conspicuous difference can be explained by social and cultural differences between these two communities like the responsibility felt by people for taking care of a disable member of the family and popularity of traditional family structures, in which sacrificing for this united union is a duty. It seems different political background of a country is closely connected to families' economic status, as well as different public health policies. Another effective factor in burden of care-giving is social support that is relative in different countries. Social support includes effective training to prepare caregivers for their new role, providing educational and occupational opportunities and may be financial supports.

This study corroborated that the majority of people with SCI were young men as it was implied in other similar studies in Iran ${ }^{5,16,19}$ and other countries. ${ }^{6,20}$ For the occupational status, we found the positive impact of the SCI individuals' employment on reducing the burden score by the caregivers. Unfortunately, a high percentage of people with SCI was unemployed. However, in cases of their employment, the burden score of caregivers was significantly lower. Perhaps, it can be implied that individuals who have a job have suffered lower level of injury, and have higher ability to perform daily activities. Accordingly, they were less dependent on their caregivers. As a result, the caregivers felt fewer burdens. In the current study, $13 \%$ of SCI people with paraplegia and $9 \%$ of those with tetraplegia injuries were employed. Furthermore, the employment of these individuals results in additional income, which can reduce the financial burden as one of the major concerns.

As it has been expected, a significant relationship was revealed between the burden score and the level of injury. Generally, tetraplegic individuals are more dependent on their caregivers for daily life activities and need more hours of care. Consequently, their caregivers endure a higher level of burden. Other similar studies indicated 
a significant correlation between the physical disability, ${ }^{1}$ the level of neurological injury, ${ }^{6}$ and the caregivers' burden. There was also no relationship between the burden of care and completeness of injury in this study.

Duration of injury and duration of care given by the current caregiver had a direct effect on the burden felt by caregivers. Perhaps, our results reflected early caregivers' anticipant of patients' recovery while they hoped that the situation is temporary. However, they later realized the permanent nature of the injury as a lifelong disability, which resulted in being frustrated and feeling more burdened. In addition, over time caregivers become older and their physical and mental ability, as well as flexibility drop.

This positive correlation between duration of SCI and burden in our study was in contrast to those studies that indicated the highest level of burden and psychological disorders, such as depression and anxiety in the immediate years after the onset of injury, and predicted the decreasing of burden after coping with new role and responsibility and situation. ${ }^{1,6,8}$ This contrast seems to be the difference in accessing proper facilities and support and training in countries.

The majority of the caregivers in our study were women (18-70 years old). It is because of women's historical sex-linked role in the family and even the society. Wives and mothers usually take the responsibility of taking care of the family members with SCI, whereas husbands or fathers have to work, have an income and are generally less directly involved in providing care for SCI individual. There was no relationship between the caregivers' gender and their burden of care in this study. Nevertheless, our study like many other similar studies ${ }^{1,6,9}$ indicates that older caregivers experience more burden because of their general ability, energy and possessing less adaptation capability for the new role compared with the younger people. These elder caregivers may suffer from some medical conditions-whether or not these difficulties are directly related to aging and also a higher care burden-which demand care and attention.

Like other studies, ${ }^{16,20}$ the current study revealed a negative correlation between the caregivers' level of education and the burden score. Although the caregivers with higher educational levels felt a lighter burden, they generally were not highly educated. Perhaps, people who spent more years going to school and even continued higher education are more capable of handling stressful situations and new roles. These individuals can improve their knowledge regarding SCI individuals, their needs and method of coping with the new situation. In addition, less educated people were typically older, and it should be noted that aging is one of the factors associated with a higher burden score.

In this study, being the only caregiver did not influence the burden. Our results revealed that caregivers who did not live with SCI individuals reported a significantly lesser burden. Despite the additional burden of commuting, the fact that these caregivers can have a private zone and solitude, even for a brief while, can benefit them to use this time to mend or reduce the physical and psychological effects of care-giving. Moreover, the individuals with SCI, who do not live with their caregivers, probably are less dependent than others who need full-time care; this fact reduces the total burden. In addition, it seems that when caregivers live with SCI individuals, the care recipients demand more services and expect their caregiver to be available all the time.

It was revealed that parents felt more burdens in comparing with the individuals' spouses. This could be explained by their age difference. Parents like to provide their children with emotional care. Nevertheless, they are older than spouses or children ${ }^{9}$ of SCI persons and the older they are, the more burdens they feel. On the other hand,
$50 \%$ of married individuals with SCI were cared for by their spouses as the main caregiver, with more men than women (53.5\% vs $38.1 \%$ ). Perhaps, men are out of house more than women because of their jobs.

We could not find any relationship between the number of SCI individuals' children and the burden score. This differs from other studies including Molazem et al., ${ }^{16}$ whose study was conducted in the Iran, and indicated that the number of children can affect the level of burden. This difference can be the result of different methods of selecting participants.

\section{Limitations}

This research was a cross-sectional study based on self-reported measures of the questionnaire. We categorized the participants in respective socioeconomic groups based on our experience, as there was no practical assessment measure, given the country's distinctive cultural and economic matters. There was a limitation in covering other variables related to the care burden and only the selected variables were examined. As an illustration, the caregiver burden should be assessed according to other psychosocial factors such as depression and social support.

\section{Implications}

The communication issues have not been covered in the questionnaire. Nevertheless, teaching communication can be implied as a mean for enhancing the patient-caregiver relationship, which results in less caregivers' burden. It is recommended to extend the current questionnaire or define a new instrument for covering these issues.

\section{CONCLUSION}

The findings of this study corroborate that the burden score of a caregiver is a multidimensional issue. It can be affected by numerous factors and these factors can be different to some extent in different countries. In the current study, the duration of SCI, level of injury and caregivers' age are variables that are significantly related to burden score. In addition, the results illustrate the significance of social factors such as care recipients' occupational status, as well as caregivers' level of education in reducing caregivers' burden. As these variables are subject to change, like employment of caregivers, it is crucial that healthcare systems identify these factors and considers them in their strategic planning for the target society.

\section{DATA ARCHIVING}

There were no data to deposit.

\section{CONFLICT OF INTEREST}

The authors declare no conflict of interest.

\section{ACKNOWLEDGEMENTS}

We acknowledge the support of the Brain and Spinal Cord Injury Research Center, Neuroscience Institute, Tehran University of Medical Sciences, Tehran, Iran. We acknowledge the Taylor Lawson, Western Washington University (WWU), Bellingham, WA, USA, for editing of our manuscript.

1 Post M, Bloemen J, De Witte L. Burden of support for partners of persons with spinal cord injuries. Spinal Cord 2005; 43: 311-319.

2 Graça Á, Nascimento MAd, Lavado EL, Garanhani MR. Quality of life of primary caregivers of spinal cord injury survivors. Rev Bras Enferm 2013; 66: 79-84.

3 Khazaeipour Z, Taheri-Otaghsara S-M, Naghdi M. Depression following spinal cord injury: its relationship to demographic and socioeconomic indicators. Top Spinal Cord Inj Rehabil 2015; 21: 149. 
4 Khazaeipour Z, Norouzi-Javidan A, Kaveh M, Khanzadeh Mehrabani F, Kazazi E, Emami-Razavi S-H. Psychosocial outcomes following spinal cord injury in Iran. J Spinal Cord Med 2014; 37: 338-345.

5 Khazaeipour Z. Effective teaching strategies for patient education in individuals with spinal cord injury in Iran. Arch Neurosci 2016; 3: e28211.

6 Nogueira PC, Rabeh SAN, Caliri MHL, Haas VJ. Caregivers of individuals with spinal cord injury: caregiver burden. Rev ESC Enferm USP 2013; 47: 607-614.

7 Middleton JW, Simpson GK, De Wolf A, Quirk R, Descallar J, Cameron ID. Psychological distress, quality of life, and burden in caregivers during community reintegration after spinal cord injury. Arch Phys Med Rehabil 2014; 95: 1312-1319.

8 Nogueira PC, Rabeh SAN, Caliri MHL, Dantas RAS, Haas VJ. Burden of care and its impact on health-related quality of life of caregivers of individuals with spinal cord injury. Rev Lat Am Enfermagem 2012; 20: 1048-1056.

9 Gajraj-Singh P. Psychological impact and the burden of caregiving for persons with spinal cord injury (SCI) living in the community in Fiji. Spinal Cord 2011; 49: 928-934.

10 Schulz R, Czaja SJ, Lustig A, Zdaniuk B, Martire LM, Perdomo D. Improving the quality of life of caregivers of persons with spinal cord injury: a randomized controlled trial. Rehabil Psychol 2009; 54: 1

11 Robison J, Fortinsky R, Kleppinger A, Shugrue N, Porter M. A broader view of family caregiving: effects of caregiving and caregiver conditions on depressive symptoms, health, work, and social isolation. J Gerontol B Psychol Sci Soc Sci 2009; 64: 788-798.
12 Rodakowski J, Skidmore ER, Rogers JC, Schulz R. Role of social support in predicting caregiver burden. Arch Phys Med Rehabil 2012; 93: 2229-2236.

13 Rajabi Mashhadi MT, Mashhadinejad H, Ebrahimzadeh MH, Golhasani-Keshtan F, Ebrahimi H, Zarei Z. The Zarit Caregiver burden interview short form (ZBI-12) in spouses of veterans with chronic spinal cord injury, validity and reliability of the persian version. Arch Bone Jt Surg 2015; 3: 56-63.

14 Blanes L, Carmagnani MIS, Ferreira L. Health-related quality of life of primary caregivers of persons with paraplegia. Spinal Cord 2007; 45: 399-403.

$15 \mathrm{Kim} \mathrm{H}$, Chang M, Rose K, Kim S. Predictors of caregiver burden in caregivers of individuals with dementia. J Adv Nurs 2012; 68: 846-855.

16 Vagharseyyedin SA, Molazem Z. Burden, resilience, and happiness in family caregivers of spinal cord injured patients. Middle EAST J Family Med 2013; 7: 29.

17 Adeosun II. Correlates of caregiver burden among family members of patients with schizophrenia in Lagos, Nigeria. Schizophr Res Treatment 2013; 2013: 353809.

$18 \mathrm{Ma} \mathrm{H-P}$, Lu H-J, Xiong X-Y, Yao J-Y, Yang Z. The investigation of care burden and coping style in caregivers of spinal cord injury patients. Int J Nurs Sci 2014; 1: 185-190.

19 Rahimi-Movaghar V, Moradi-Lakeh M, Rasouli MR, Vaccaro A. Burden of spinal cord injury in Tehran, Iran. Spinal Cord 2010; 48: 492-497.

20 Raj J, Manigandan C, Jacob K. Leisure satisfaction and psychiatric morbidity among informal carers of people with spinal cord injury. Spinal Cord 2006; 44: 676-679. 